\title{
Qualidade dos sedimentos no rio Uberabinha (Uberlândia, MG) e implicações ambientais
}

\author{
Vania Rosolen ${ }^{1}$ Uwe Herpin ${ }^{2}$, Nivia Maria Melo Coelho ${ }^{3}$, Luciana Melo Coelho ${ }^{4}$, \\ Jorge Luis Silva Brito ${ }^{5}$, Luciano Alves da Silva ${ }^{6} \&$ Samuel do Carmo Lima ${ }^{7}$
}

\begin{abstract}
Resumo Os sedimentos são constituídos por partículas minerais (areia, silte e argila) e matéria orgânica que são transportadas ou depositadas nos corpos d'água. Atividades antrópicas como industrialização e urbanização são responsáveis pela entrada de altas cargas de elementos tóxicos nas águas superficiais das bacias hidrográficas, e podem afetar negativamente a qualidade dos sedimentos. Os sedimentos podem estocar substâncias perigosas ou, então, atuar como uma fonte secundária de poluição através da liberação de contaminantes no sistema. O objetivo deste artigo é avaliar a concentração de elementos tóxicos em sedimentos coletados no Rio Uberabinha (Uberlândia, MG) que pode representar um risco para o meio ambiente e saúde humana.

A análise química dos sedimentos em diferentes pontos do rio Uberabinha indicou elevada concentração de alguns metais, como por exemplo, $\mathrm{Co}, \mathrm{Cu}, \mathrm{Ni}, \mathrm{Pb}$ e $\mathrm{Zn}$, relacionados com a descarga de efluentes urbanos (industrial e doméstico) sem tratamento nos corpos d'água.
\end{abstract}

Palavras-chave: bacia hidrográfica, qualidade dos sedimentos, elementos tóxicos, poluição.

\begin{abstract}
Quality of sediments and environmental implications in the Uberabinha River (Uberlândia, $M G$ ). Sediments are constituted of mineral particles (sand, silt and clay) and organic matter transported or deposited in the water bodies. Anthropogenic activities as industrialization and urbanization are responsible for the input of high amounts of toxic metals to the surface water of a River Basin and can negatively impact sediment quality. The sediments can storage hazardous substances or act as a secondary pollution source by transfering contaminants to the system. The objective of this study was to evaluate the concentration of toxic elements in sediments of Uberabinha River (Uberlândia, MG) which may represent a risk for the environment and human health. The Chemical analysis of sediments at different localities indicated elevated concentration of some metals, e.g., $\mathrm{Co}, \mathrm{Cr}, \mathrm{Cu}, \mathrm{Ni}, \mathrm{Pb}$ and $\mathrm{Zn}$ compared to guiding values for sediment quality established by the National Environment Council (CONAMA, Brazil). and could be related to the direct discharge of untreated urban (industrial and domestic) effluents into the water stream.
\end{abstract}

Keywords: river basin, sediments quality, toxic elements, pollution.

INTRODUÇÃ̃O Os sedimentos fluviais são partes integrais, essenciais e dinâmicas das bacias hidrográficas e possuem valor ambiental, social e econômico (Network 2007). A quantidade e qualidade dos sedimentos têm importância fundamental para manter o adequado equilíbrio e funcionamento dos rios.

Os estudos relacionados aos sedimentos focam os aspectos quantitativo e qualitativo e ambos têm impacto sobre as condições ecológico-ambientais de uma bacia hidrográfica (Salomons \& Brils, 2004). Considerando os aspectos quantitativos, mudanças hidrológicas influenciadas pelo clima e pelo homem podem modificar o tempo, a intensidade e a descarga disponível efe- tiva para o transporte dos sedimentos (Vörösmarty et al., 2003). Por exemplo, estima-se que apenas com a construção dos grandes reservatórios ao redor do mundo são interceptadas mais de $40 \%$ da descarga global da água e que, aproximadamente, de 25 a 30\% (4-5 Gt/ano) do fluxo de sedimentos que seriam transportados para a costa ficam retidos em barragens (Brismar, 2002). Quanto aos aspectos qualitativos, estes estão relacionados à presença de contaminantes que entram no sistema fluvial por diferentes caminhos, através dos meios rural e urbano (erosão do solo, desmatamentos, lixiviação dos materiais de construção e dos sistemas de esgoto etc), por meio de fontes diretas ou difusas.

1 - Universidade Federal de Uberlândia, Profa. Dra. do Instituto de Geografia, Minas Gerais, Brasil. E-mail: vrosolen@ig.ufu.br

2 - Universidade de São Paulo, Pesquisador do Núcleo de Pesquisa em Geoquímica e Geofísica da Litosfera, Piracicaba (SP), Brasil.

E-mail: uwe.herpin@gmail.com

3 - Universidade Federal de Uberlândia, Profa. Dra. do Instituto de Química, Uberlândia (MG), Brasil. E-mail: nmmcoelho@ufu.br

4 - Universidade Federal de Goiás, Profa. Dra. do Departamento de Química, Catalão (GO), Brasil. E-mail: lucianamelo71@click21.com.br

5 - Universidade Federal de Uberlândia, Prof. Dr. do Instituto de Geografia, Uberlândia (MG), Brasil. E-mail: jlbrito@ufu.br

6 - Universidade Federal de Uberlândia, Mestrando do Programa de Pós-Graduação em Química, Uberlândia (MG), Brasil.

E-mail: luciano tj@yahoo.com.br

7 - Universidade Federal de Uberlândia, Prof. Dr. do Instituto de Geografia, Uberlândia (MG), Brasil. E-mail: samuel@ufu.br 
Os sedimentos são reservatórios de elementostraço biodisponíveis que ficam retidos nos minerais por processos de adsorção, precipitação, oclusão e incorporação (Ahlf \& Förstner, 2001). A contaminação com elementos-traço de origem antropogênica é um persistente problema nas sociedades industriais pois estes poluentes são não-degradáveis e se acumulam em formas químicas que são, muitas vezes, mais reativas que as formas originais (Adriano et al., 2004). Sedimentos contaminados estão presentes em várias áreas de deposição como portos, lagos, barragens e planícies de inundação (Salomons, 2005).

Devido à capacidade que os sedimentos têm em estocar e imobilizar elementos tóxicos eles podem ser considerados como "armadilhas geoquímicas" e os efeitos diretos da poluição podem não se manifestar imediatamente. Embora esta função possa ser considerada positiva, não há garantia de que os elementos químicos estarão segura e indefinidamente estocados (Stigliani, 1988; Förstner, 2003). Fatores ambientais que influenciam na capacidade de estocagem dos sedimentos ou na biodisponibilidade dos elementos estocados, podem mudar e indiretamente causar a rápida e inesperada mobilização dos elementos químicos no ambiente. $\mathrm{O}$ mais desastroso resultado, segundo Förstner (2003), é quando nos períodos de inundação, poluentes ligados aos sedimentos ultrapassam os limites das planícies de inundação e invadem áreas residenciais e agrícolas.

Vários estudos realizados no Brasil apontaram sérios problemas de contaminação de sedimentos e alta biodisponibilidade potencial de metais em reservatórios e segmentos de rios a jusante de grandes centros urbanos (Robaina et al., 2002, Mozeto et al., 2003, Hissler et al., 2004, Kasper et al., 2004).

Este artigo apresenta os resultados das concentrações químicas totais obtidas em sedimentos do Rio Uberabinha. As atividades agrícolas predominam na bacia, com uso intensivo de fertilizantes e pesticidas (não raro aplicados por avião), extração de argila refratária próximo à cabeceira do rio, urbanização e industrialização do município de Uberlândia e, finalmente, a presença de lagos e reservatórios para geração de energia. O relatório oficial gerado pelo IGAM (Instituto Mineiro de Gestão das Águas, 2005) determinou índices críticos de poluição e qualidade das águas em determinados segmentos da bacia.

O objetivo deste estudo foi avaliar a concentração de elementos potencialmente tóxicos nos sedimentos recentes depositados em alguns segmentos do Rio Uberabinha (Uberlândia, MG) visando entender os efeitos das atividades humanas sobre este recurso que podem representar um risco para o meio ambiente e saúde humana. É importante salientar que estes são os resultados iniciais de um projeto de pesquisa piloto uma vez que não havia qualquer informação anterior sobre a qualidade dos sedimentos dos rios da região.

\section{MATERIAIS E MÉTODOS}

Localização da área $\mathrm{O}$ rio Uberabinha é um tributário da Bacia do Rio Araguari. Possui extensão de aproximadamente $140 \mathrm{~km}$ sendo que, ao meio de seu curso $(80 \mathrm{~km})$, localiza-se a cidade de Uberlândia (Triângulo Mineiro, MG) (Fig. 1). A escolha deste rio para o desenvolvimento da pesquisa deve-se ao fato de que suas águas são usadas para o abastecimento público (a população urbana é de aproximadamente 700.000 habitantes), para irrigação (cana-de-açúcar, soja, milho e pastagem) e geração de energia.

O clima regional é tropical, caracterizado por um período marcadamente seco (entre maio e outubro) e outro úmido (entre novembro e abril). A precipitação média anual é de $1800 \mathrm{~mm}$ e a temperatura média anual é de aproximadamente $23^{\circ} \mathrm{C}$. A geologia é representada pelos arenitos das Formações Marília e Botucatu e pelos basaltos da Formação Serra Geral. A topografia é predominantemente caracterizada por platôs com superfícies planas (chapadas) e a vegetação original é o Cerrado, quase todo substituído pela agricultura e pastagem. Na região predominam os solos lateríticos, com argilas de baixa atividade como caulinita, óxidos de ferro (hematita) e de alumínio (gibsita), baixo $\mathrm{pH}$ e teor de carbono, exceto nas áreas de veredas (solos hidromórficos).

O monitoramento da qualidade das águas do rio é realizado desde 1998 pelo IGAM (Instituto Mineiro de Gestão das Águas), porém não há pontos de coleta de sedimentos para avaliação de parâmetros qualitativos. O rio Uberabinha é um afluente do Rio Araguarí e está inserido na Unidade de Planejamento e Gestão de Recursos Hídricos (UPGRH) PN2, que corresponde a Bacia do Paranaíba. No rio Uberabinha o IGAM opera dois pontos de monitoramento da qualidade das águas. Um ponto de monitoramento está instalado a montante e o outro ponto está localizado a jusante da cidade de Uberlândia. O último relatório sobre o Índice de Qualidade das Águas (IQA) superficiais divulgado pelo órgão em 2006 (INSTITUTO MINEIRO DE GESTÃO DAS ÁGUAS, inédito) apontou elevadas concentrações de coliformes fecais, de fósforo e contaminação por elementos tóxicos relacionados à descarga de esgoto doméstico e industrial provenientes da cidade de Uberlândia.

\section{Amostragem do sedimento, preparação das amos-} tras e análises de laboratório Por se tratar de um estudo piloto, foram escolhidos pontos de amostragem representativos dos diferentes usos do solo da bacia, que permitiram a caracterização preliminar e geral sobre os níveis de concentração de elementos químicos nos sedimentos recentemente depositados, uma vez que não havia informações prévias sobre a qualidade dos sedimentos no rio ou da bacia. As amostras foram coletadas nas margens do rio, próximo às planícies de inundação, em locais de baixa energia fluvial. Em cinco pontos ao longo de um segmento do Uberabinha (Fig. 1) foram amostrados sedimentos recentemente depositados: (1) a montante da cidade de Uberlândia, em um dos reservatórios de água para abastecimento público (reservatório de Sucupira); (2) área densamente urbanizada próximo ao ponto de lançamento de esgoto doméstico; (3) ponto 
localizado no tributário córrego Liso que drena o distrito industrial, aproximadamente $25 \mathrm{~m}$ antes da confluência com o rio Uberabinha; (4) confluência do córrego Liso com rio Uberabinha e (5) ponto mais a jusante no rio Uberabinha.

As amostras foram coletadas entre $0-10 \mathrm{~cm}$ de profundidade. Os sedimentos foram homogeneizados, secados em estufa entre $50-60^{\circ} \mathrm{C}$, misturados, peneirados em peneira com malha de nylon para obter a fração $<0,062 \mathrm{~mm}$, seguindo os procedimentos propostos por Mudrock \& Mcknight (1994) citado por Förstner (2004). Em cada ponto foram coletadas três amostras para calculo da média e desvio padrão.

O Carbono Total foi determinado por Infra-Vermelho LECO, modelo CS-225, no Laboratório Lakefield-Geosol.

A determinação dos óxidos $\mathrm{SiO}_{2}, \mathrm{Al}_{2} \mathrm{O}_{3}, \mathrm{TiO}_{2}$, $\mathrm{Fe}_{2} \mathrm{O}_{3}, \mathrm{MnO}, \mathrm{MgO}, \mathrm{CaO}, \mathrm{Na}_{2} \mathrm{O}, \mathrm{K}_{2} \mathrm{O}$ e $\mathrm{P}_{2} \mathrm{O}_{5}$ foi por Espectroscopia de Raios-X (Laboratório Lakefield-Geosol).

A análise multielementar para a determinação de 21 elementos (As, Ba, Cd, Co, Cr, Cu, La, Li, Mn, $\mathrm{Mo}, \mathrm{Ni}, \mathrm{Pb}, \mathrm{Sb}, \mathrm{Sc}, \mathrm{Sn}, \mathrm{Sr}$, Ti, V, W, Y e Zn) foi realizada por ICP-OES, Thermo Jarrel Ash, ICAP 61E, após digestão das amostras com água régia $\left(\mathrm{HCl}-\mathrm{HNO}_{3}\right.$, $3: 1)$. O controle de qualidade foi verificado usando o material de referência interno (ICPREF20, Laboratório Lakefield-Geosol LTDA, Brasil).

RESULTADOS E DISCUSSÕES Os sedimentos fluviais resultam da erosão da rocha e do solo presentes na bacia (Owens, 2005). Desta forma, a composição química dos sedimentos reflete parcialmente estes materiais (Förstner et al., 2004). Os teores de óxidos determinados nas amostras dos sedimentos são compatíveis com as áreas fontes, constituídas pelos solos lateríticos (Latossolos e Argissolos) e produtos da alteração das rochas areníticas e basálticas que se distribuem por toda a bacia que quando erodidos e transportados depositamse na calha fluvial. São compostos majoritariamente por $\mathrm{SiO}_{2}$ (valores determinados entre 69,4 e 77,8 \%), $\mathrm{Al}_{2} \mathrm{O}_{3}$ (entre 4,9 e $10,9 \%$ ) e $\mathrm{Fe}_{2} \mathrm{O}_{3}$ (entre 3,5 e $10,4 \%$ ), e apresentaram baixas concentrações relativas de $\mathrm{CaO}$ (entre 0,1 e $1,3 \%$ ), $\mathrm{K}_{2} \mathrm{O}$ (entre 0,1 e $0,3 \%$ ), $\mathrm{MgO}$ (entre 0,2 e $0,5 \%), \mathrm{MnO}^{2}(0,1 \%)$ e $\mathrm{P}_{2} \mathrm{O}_{5}$ (entre 0,1 e $0,2 \%$ ) (Tab. 1). As concentrações mais elevadas de $\mathrm{Fe}_{2} \mathrm{O}_{3}$ foram determinadas nos sedimentos coletados nos Pontos 5,3 e 4, respectivamente. Nestes segmentos do córrego Liso e do rio Uberabinha o assoreamento nas planícies de inundação é evidente e está associado ao acelerado processo de erosão das margens e desprovidas de vegetação ciliar. O material laterítico depositado continuamente contribuiu com estes resultados pois teores elevados de ferro e alumínio são próprios da constituição químico-mineralógica dos solos tropicais fortemente intemperizados como aqueles que desenvolvidos no município de Uberlândia (Wilcke et al., 1999).

$\mathrm{O}$ teor de macroelementos $\mathrm{K}, \mathrm{Mg}, \mathrm{Ca}$ e $\mathrm{Na}$ (Tab. 2) é baixo em todos os pontos (sempre inferior a $0,2 \%$ ) exceto por um ligeiro aumento no Ponto 2, possivelmente relacionado à disposição do esgoto domés-

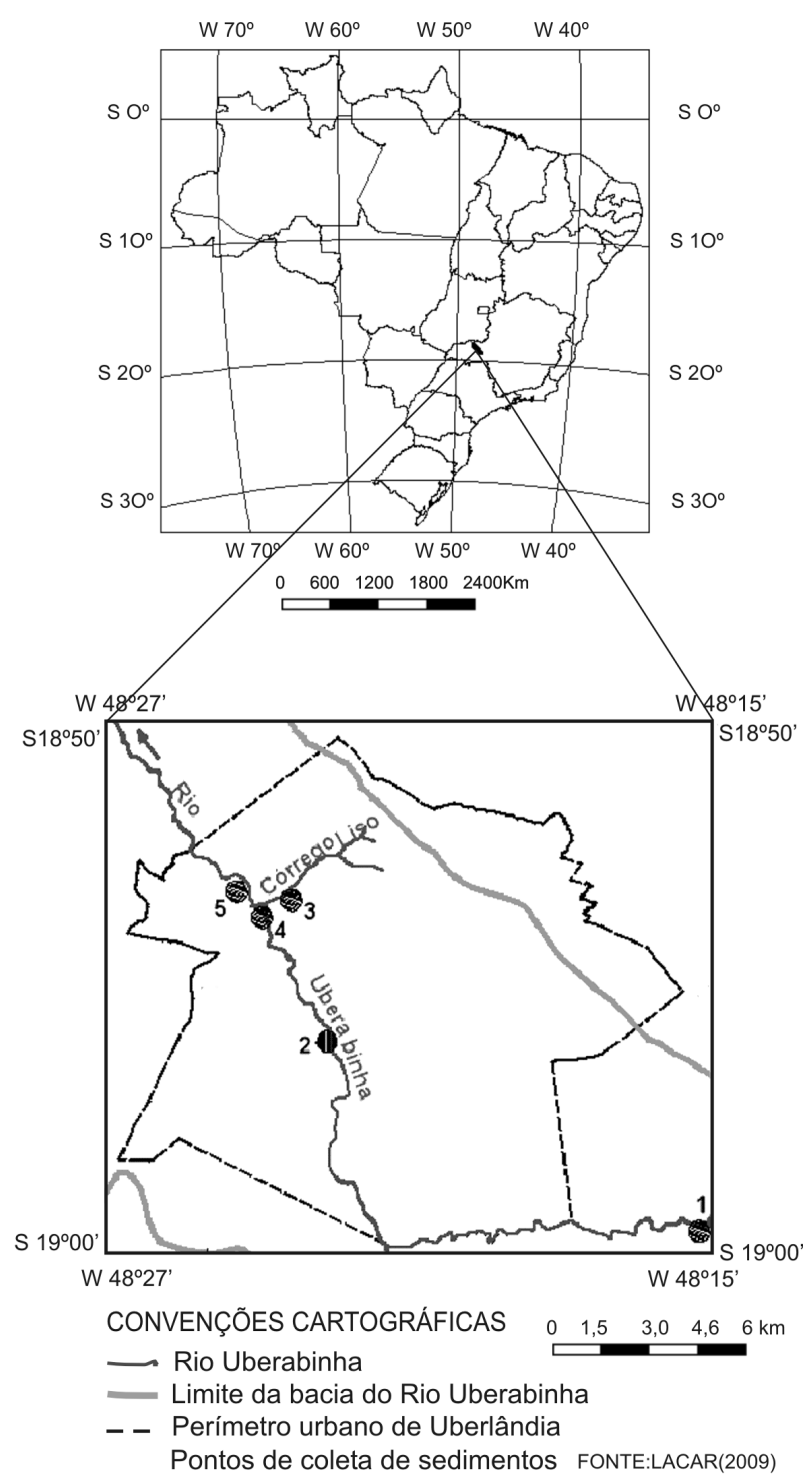

Figura 1 - Localização da área de trabalho.

tico. O esgoto doméstico contém quantidades significativas de matéria orgânica, macro e micronutrientes e elementos poluentes (Rosolen et al., 2005). A disposição deste material nos corpos hídricos é uma prática comum nos municípios brasileiros e pode resultar em eutrofização das águas e contaminação com patógenos e elementos tóxicos. Em relação ao carbono total (Tab. 2) duas situações podem refletir no ligeiro aumento da concentração. A primeira situação, no Ponto 2 , o teor varia entre 1,4 e $1,5 \%$ e pode estar associado ao maior despejo de esgoto doméstico e, a segunda situação, no Ponto 1 , com teor entre 1,2 e $1,3 \%$, pode estar relacionado com a presença da matéria orgânica da vegetação ciliar, relativamente mais bem preservada nesta área. Não foi identificado aumento considerável de fósforo associado à disposição do esgoto doméstico.

Quanto à presença de elementos tóxicos nos sedimentos (Tab. 3), os resultados da análise multielementar por ICP, revelaram altas concentrações que refletem a influência direta e/ou indireta dos efeitos da 
Tabela 1 - Teor dos óxidos determinados nos pontos de coleta dos sedimentos depositados no Rio Uberabinha, Uberlândia $(M G)$.

\begin{tabular}{|c|c|c|c|c|c|c|c|c|c|}
\hline \multirow[t]{2}{*}{ Pontos de Coleta } & \multicolumn{9}{|c|}{ Óxidos (\%) } \\
\hline & $\mathrm{Al}_{2} \mathrm{O}_{3}$ & $\mathrm{CaO}$ & $\mathrm{Fe}_{2} \mathrm{O}_{3}$ & $\mathrm{~K}_{2} \mathrm{O}$ & $\mathrm{MgO}$ & $\mathrm{MnO}$ & $\mathrm{Na}_{2} \mathrm{O}$ & $\mathrm{P}_{2} \mathrm{O}_{5}$ & $\mathrm{SiO}_{2}$ \\
\hline P1.1 & 10,7 & 0,1 & 3,5 & 0,0 & $<0.1$ & 0,0 & $<0.1$ & 0,1 & 74,9 \\
\hline P1.2 & 10,6 & 0,0 & 4,0 & 0,0 & $<0.1$ & 0,0 & $<0.1$ & 0,1 & 75,0 \\
\hline $\mathrm{P} 1.3$ & 11,5 & 0,0 & 3,0 & 0,0 & $<0.1$ & 0,0 & $<0.1$ & 0,1 & 73,2 \\
\hline Média & 10,9 & 0,1 & 3,5 & 0,0 & - & 0,0 & - & 0,1 & 74,4 \\
\hline DP & 0,5 & 0,0 & 0,5 & 0,0 & - & 0,0 & - & 0,0 & 1,0 \\
\hline $\mathrm{P} 2.1$ & 10,3 & 1,4 & 8,6 & 0,3 & 0,5 & 0,1 & 0,1 & 0,2 & 68,4 \\
\hline $\mathrm{P} 2.2$ & 9,7 & 1,3 & 8,3 & 0,2 & 0,5 & 0,1 & 0,1 & 0,2 & 70,0 \\
\hline $\mathrm{P} 2.3$ & 10,1 & 1,3 & 8,2 & 0,3 & 0,5 & 0,1 & 0,1 & 0,2 & 69,8 \\
\hline Média & 10,0 & 1,3 & 8,4 & 0,3 & 0,5 & 0,1 & 0,1 & 0,2 & 69,4 \\
\hline DP & 0,3 & 0,1 & 0,2 & 0,0 & 0,0 & 0,0 & 0,0 & 0,0 & 0,9 \\
\hline P3.1 & 6,1 & 0,2 & 9,8 & 0,1 & 0,2 & 0,1 & $<0.1$ & 0,1 & 76,4 \\
\hline $\mathrm{P} 3.2$ & 6,1 & 0,2 & 9,3 & 0,1 & 0,2 & 0,1 & $<0.1$ & 0,1 & 76,0 \\
\hline P3.3 & 6,0 & 0,2 & 8,9 & 0,1 & 0,2 & 0,1 & $<0.1$ & 0,1 & 77,4 \\
\hline Média & 6,1 & 0,2 & 9,3 & 0,1 & 0,2 & 0,1 & - & 0,1 & 76,6 \\
\hline DP & 0,1 & 0,0 & 0,5 & 0,0 & 0,0 & 0,0 & - & 0,0 & 0,7 \\
\hline P4.1 & 5,0 & 0,2 & 9,5 & 0,1 & 0,1 & 0,1 & $<0.1$ & 0,1 & 77,8 \\
\hline $\mathrm{P} 4.2$ & 4,9 & 0,2 & 9,3 & 0,1 & 0,2 & 0,1 & $<0.1$ & 0,1 & 77,7 \\
\hline P4.3 & 4,9 & 0,2 & 9,2 & 0,1 & 0,2 & 0,1 & $<0.1$ & 0,1 & 78,0 \\
\hline Média & 4,9 & 0,2 & 9,3 & 0,1 & 0,2 & 0,1 & - & 0,1 & 77,8 \\
\hline DP & 0,1 & 0,0 & 0,2 & 0,0 & 0,0 & 0,0 & - & 0,0 & 0,2 \\
\hline P5.1 & 8,8 & 0,2 & 10,7 & 0,1 & 0,1 & 0,1 & $<0.1$ & 0,2 & 70,9 \\
\hline P5.2 & 8,8 & 0,2 & 10,2 & 0,1 & 0,2 & 0,1 & $<0.1$ & 0,2 & 70,8 \\
\hline P5.3 & 8,9 & 0,2 & 10,4 & 0,1 & 0,2 & 0,1 & $<0.1$ & 0,2 & 69,8 \\
\hline Média & 8,8 & 0,2 & 10,4 & 0,1 & 0,2 & 0,1 & - & 0,2 & 70,5 \\
\hline DP & 0,1 & 0,0 & 0,3 & 0,0 & 0,0 & 0,0 & - & 0,0 & 0,6 \\
\hline
\end{tabular}

DP: Desvio padrão calculado para 3 medidas.

urbanização e das atividades humanas. Há histórico de alta concentração e alta biodisponibilidade de $\mathrm{Cd}, \mathrm{Cu}$, $\mathrm{Mn}, \mathrm{Ni}, \mathrm{Pb}$ e Zn nos solos urbanos de Uberlândia (Wilcke et al., 1999) sendo que as maiores concentrações de $\mathrm{Cu}, \mathrm{Mn}, \mathrm{Ni}$ e $\mathrm{Zn}$ ocorreram nos solos do setor industrial. A maior concentração de $\mathrm{Pb}$ foi encontrados no setor Sul, próximo ao centro, provavelmente relacionado ao intenso tráfego de veículos próximo ao centro. Emissão veicular nas cidades é responsável por importante parte da poluição antropogênica com metais traço (Miguel, 1992). Apesar da proibição do uso de antidetonantes a base de chumbo nos veículos no final da década de 1980, ainda se usa este elemento como aditivo na gasolina (Miguel, 1999; Duarte \& Pasqual, 2000; Carneiro, 2004). As fontes de Cd não foram identificadas. Hou- ve significantes correlações entre o teor de carbono e a concentração de $\mathrm{Al}(\mathrm{r}=0,63), \mathrm{Cr}(\mathrm{r}=0,50), \mathrm{Ni}(\mathrm{r}=$ $0,58)$ e $\mathrm{Pb}(\mathrm{r}=0,56)$ e entre óxidos de $\mathrm{Fe}$ (extraível com ácido ascórbico) e o total de concentração de todos os metais $(\mathrm{r}=0,51$ a 0,94$)$, exceto $\mathrm{Pb}$. Os resultados ressaltando a importância da matéria orgânica e dos óxidos de ferro para o estoque de poluentes nos solos lateríticos fortemente alterados.

Considerando os 21 elementos analisados nesta pesquisa (Tab. 3), As, Cd, Mn, Mo, Sb, Sn e W não estão presentes nos sedimentos ou estão fora do limite de detecção instrumental. Comparando os resultados obtidos nos cinco pontos de amostragem, os sedimentos coletados no Ponto 1 (reservatório de Sucupira) apresentaram elevadas concentrações médias de $\mathrm{La}(72,3 \mathrm{mg} /$ 
Tabela 2 - Teor dos macroelementos determinados nos pontos de coleta dos sedimentos do rio Uberabinha, Uberlândia $(M G)$.

\begin{tabular}{|c|c|c|c|c|c|c|}
\hline \multirow{2}{*}{$\begin{array}{c}\text { Pontos de } \\
\text { Coleta }\end{array}$} & \multicolumn{6}{|c|}{ Elementos (\%) } \\
\hline & $\mathrm{C}$ & $\mathrm{P}$ & $\mathrm{Ca}$ & $\mathrm{K}$ & $\mathrm{Mg}$ & $\mathrm{Na}$ \\
\hline P1.1 & 1,3 & 0,0 & 0,1 & 0,0 & 0,0 & 0,2 \\
\hline $\mathrm{P} 1.2$ & 1,2 & 0,0 & 0,0 & 0,0 & 0,0 & 0,1 \\
\hline P1.3 & 1,2 & 0,0 & 0,1 & 0,0 & 0,0 & 0,1 \\
\hline Média & 1,2 & 0,0 & 0,1 & 0,0 & 0,0 & 0,1 \\
\hline DP & 0,1 & 0,0 & 0,0 & 0,0 & 0,0 & 0,1 \\
\hline $\mathrm{P} 2.1$ & 1,5 & 0,1 & 0,9 & 0,2 & 0,3 & 0,2 \\
\hline P2.2 & 1,4 & 0,1 & 0,8 & 0,2 & 0,3 & 0,2 \\
\hline P2.3 & 1,5 & 0,1 & 0,8 & 0,2 & 0,3 & 0,2 \\
\hline Média & 1,5 & 0,1 & 0,8 & 0,2 & 0,3 & 0,2 \\
\hline DP & 0,1 & 0,0 & 0,0 & 0,0 & 0,0 & 0,0 \\
\hline P3.1 & 0,5 & 0,1 & 0,1 & 0,1 & 0,1 & 0,0 \\
\hline $\mathrm{P} 3.2$ & 0,5 & 0,1 & 0,1 & 0,1 & 0,1 & 0,0 \\
\hline P3.3 & 0,5 & 0,1 & 0,2 & 0,1 & 0,1 & 0,0 \\
\hline Média & 0,5 & 0,1 & 0,2 & 0,1 & 0,1 & 0,0 \\
\hline $\mathrm{DP}$ & 0,0 & 0,0 & 0,0 & 0,0 & 0,0 & 0,0 \\
\hline P4.1 & 0,5 & 0,0 & 0,1 & 0,1 & 0,1 & 0,0 \\
\hline $\mathrm{P} 4.2$ & 0,5 & 0,0 & 0,1 & 0,1 & 0,1 & 0,0 \\
\hline P4.3 & 0,5 & 0,0 & 0,1 & 0,1 & 0,1 & 0,0 \\
\hline Média & 0,5 & 0,0 & 0,1 & 0,1 & 0,1 & 0,0 \\
\hline $\mathrm{DP}$ & 0,0 & 0,0 & 0,0 & 0,0 & 0,0 & 0,0 \\
\hline P5.1 & 0,6 & 0,1 & 0,1 & 0,1 & 0,1 & 0,1 \\
\hline $\mathrm{P} 5.2$ & 0,6 & 0,1 & 0,1 & 0,1 & 0,1 & 0,1 \\
\hline P5.3 & 0,6 & 0,1 & 0,1 & 0,1 & 0,1 & 0,0 \\
\hline Média & 0,6 & 0,1 & 0,1 & 0,1 & 0,1 & 0,1 \\
\hline DP & 0,0 & 0,0 & 0,0 & 0,0 & 0,0 & 0,0 \\
\hline
\end{tabular}

DP: Desvio padrão calculado para 3 medidas.

$\mathrm{kg}), \mathrm{Li}(9,3 \mathrm{mg} / \mathrm{kg}), \mathrm{Ni}(40,3 \mathrm{mg} / \mathrm{kg}), \mathrm{Pb}(56,7 \mathrm{mg} / \mathrm{kg})$ e Y $(32,0 \mathrm{mg} / \mathrm{kg})$. No Ponto 2 (área densamente urbanizada) foram determinadas altas concentrações médias de $\mathrm{Ba}(121,7 \mathrm{mg} / \mathrm{kg}), \mathrm{Sr}(54,3 \mathrm{mg} / \mathrm{kg})$ e Zn $(133,3 \mathrm{mg} /$ $\mathrm{kg}$ ). No Ponto 3 (córrego Liso que drena o distrito industrial, a $25 \mathrm{~m}$ da confluência com o rio Uberabinha) nenhum elemento foi determinado em concentrações médias mais elevadas e, no Ponto 4 (confluência entre o córrego Liso e Uberabinha) houve maior concentração de $\mathrm{Cr}(137,0 \mathrm{mg} / \mathrm{kg})$. Finalmente, o Ponto 5 (jusante da cidade de Uberlândia) apresentou elevadas concentrações médias dos elementos Co $(50,3 \mathrm{mg} / \mathrm{kg}), \mathrm{Cu}(103,7$ $\mathrm{mg} / \mathrm{kg}), \mathrm{Sc}(16,7 \mathrm{mg} / \mathrm{kg})$, Ti $(2,4 \mathrm{mg} / \mathrm{kg})$ e V $(314,7 \mathrm{mg} /$ $\mathrm{kg})$. As concentrações máximas e mínimas totais dos elementos estão apresentadas na tabela 3.

Os valores obtidos apontaram a influência das atividades antropogênicas na bacia. Contudo, os valores obtidos neste trabalho não indicaram efeito acumulativo de elementos tóxicos de montante para jusante como obtidos e apresentados, por exemplo, por Singh et al. (1997) na India (rio Gomati) e por Förstner et al. (2004) na Alemanha (rio Elba)., apesar da descarga de esgoto doméstico e industrial pouco ou parcialmente tratado nas águas.

No início da pesquisa, o ponto mais a montante - Ponto 1 no lago do reservatório de Sucupira - seria considerado como representativo de valores de referência ou valores naturais de background devido à sua distância da área urbana, situada a jusante, e pela presença da mata ciliar relativamente preservada no entorno. Porém, as amostras coletadas apresentaram altas concentrações de $\mathrm{Cr}, \mathrm{Cu}, \mathrm{La}, \mathrm{Li}, \mathrm{Ni}, \mathrm{Pb}, \mathrm{Sc}, \mathrm{V}$ e Y. Particularmente neste local, por ser área de coleta de água para abastecimento público, a situação pode ser considerada crítica se os sedimentos representarem uma fonte secundária de poluição das águas e se houver a liberação e biodisponibilidade destes metais (Förstner et al., 2004, Adriano et al., 2004). Esgotos industriais, mineração e escoamento superficial urbano são reconhecidos como as principais fontes destes elementos (Adriano et al., 2004; Owens, 2005). Adicione-se a este grupo os insumos agrícolas ou subprodutos usados como fertilizantes e corretivos na agricultura, nos quais são adicionados importante quantidade de metais pesados (dados publicados há 20 anos indicaram a adição global de 30.000 a $250.000 \mathrm{~kg}^{\mathrm{ano}} \mathrm{-}^{-1} \mathrm{de} \mathrm{Cd}$, de 50.000 a $580.000 \mathrm{~kg} \mathrm{ano}^{-1}$ de $\mathrm{Cu}$, de 200.000 a $550.000 \mathrm{~kg}$ ano $^{-1}$ de Ni, de 260.000 a $1.100 .000 \mathrm{~kg}^{2}$ ano $^{-1}$ de $\mathrm{Zn}$ e de 420.000 a $2.300 .000 \mathrm{~kg} \mathrm{ano}^{-1} \mathrm{de} \mathrm{Pb}$ ) (Nriagu \& Pacyna, 1988). Metais pesados como sais de $\mathrm{Zn}$, arsenatos de $\mathrm{Cu}$ e $\mathrm{Pb}$ e compostos metalo-orgânicos normalmente fazem parte de princípios ativos de vários agroquímicos (Alloway, 1990; Angelotti-Neto et al., 2004; Owens, 2005). A alteração dos valores naturais de metais pesados nos solos também pode estar relacionada ao uso de fertilizantes fosfatados. No caso das rochas fosfatadas e apatíticas extraídas ao sul de Minas Gerais e usadas na agricultura, foram extraídos $8,7 \mathrm{mg} \mathrm{kg}^{-1}$ de $\mathrm{Cd}$, entre $81 \pm 5 \mathrm{mg} \mathrm{kg}^{-1}$ a $215 \pm 19 \mathrm{mg} \mathrm{kg}^{-1}$ de $\mathrm{Pb}$, entre $4,2 \pm 0,2$ a $115 \pm 0,5 \mathrm{mg} \mathrm{kg}^{-1}$ de $\mathrm{Cu}$ além de teores variáveis de $\mathrm{Zn}$, Cr e Ni (Campos et al., 2005). Os agrotóxicos também contribuem para o aumento da concentração de metais no ambiente pois, apesar de constituírem em uma pequena porcentagem dos poluentes totais, seu impacto é considerável devido à toxicidade e bioacumulação (Silva \& Fay, 2004). Na bacia do Uberabinha, especialmente nas chapadas onde se localizam as nascentes, o uso do solo está ligado às monoculturas de grãos (soja e milho, principalmente) para mercado externo e a canade-açúcar que vem ganhando espaço nos últimos anos. Todo o solo do entorno da represa de Sucupira é usado para agricultura e, secundariamente, por pastagem e, portanto, estas atividades devem ser consideradas possíveis fontes difusas de poluentes. O quadro é agravado pela retirada quase completa das APPs (matas ciliares, vegetação de veredas e zonas úmidas características das fitofisionomias do cerrado) e erosão superficial ou em sulcos em solos agrícolas degradados. Por outro lado, as águas mais calmas da represa favorecem o aumento da retenção e estoque dos elementos devido ao maior tempo de contato entre as fases líquida e sólida (Hissler et al.). A composição química das rochas e produtos de 
Tabela 3 - Teor dos elementos determinados nos pontos de coleta dos sedimentos do rio Uberabinha, Uberlândia $(M G)$. Elementos organizados em ordem alfabética.

\begin{tabular}{|c|c|c|c|c|c|c|c|c|c|c|c|c|c|c|}
\hline \multirow{2}{*}{$\begin{array}{l}\text { Pontos de } \\
\text { Coleta }\end{array}$} & \multicolumn{14}{|c|}{ Elementos (mg/kg) } \\
\hline & $\mathrm{Ba}$ & $\mathrm{Co}$ & $\mathrm{Cr}$ & $\mathrm{Cu}$ & $\mathrm{La}$ & $\mathrm{Li}$ & $\mathrm{Mn}$ & $\mathrm{Ni}$ & $\mathrm{Pb}$ & $\mathrm{Sc}$ & $\mathrm{Sr}$ & $\mathrm{Ti}$ & V & $\mathrm{Zr}$ \\
\hline $\mathrm{P} 1.1$ & 39,0 & 33,0 & 104,0 & 74,0 & 71,0 & 8,9 & $<0.01$ & 40,0 & 64,0 & 16,0 & 15,0 & 2,0 & 156,0 & 174,0 \\
\hline $\mathrm{P} 1.2$ & 37,0 & 31,0 & 105,0 & 75,0 & 70,0 & 8,9 & $<0.01$ & 41,0 & 50,0 & 16,0 & 13,0 & 1,8 & 145,0 & 168,0 \\
\hline $\mathrm{P} 1.3$ & 39,0 & 34,0 & 108,0 & 76,0 & 76,0 & 10,0 & $<0.01$ & 40,0 & 56,0 & 17,0 & 13,0 & 1,9 & 153,0 & 186,0 \\
\hline Média & 38,3 & 32,7 & 105,7 & 75,0 & 72,3 & 9,3 & - & 40,3 & 56,7 & 16,3 & 13,7 & 1,9 & 151,3 & 176,0 \\
\hline DP & 1,2 & 1,5 & 2,1 & 1,0 & 3,2 & 0,6 & - & 0,6 & 7,0 & 0,6 & 1,2 & 0,1 & 5,7 & 9,2 \\
\hline $\mathrm{P} 2.1$ & 126,0 & 26,0 & 69,0 & 72,0 & 24,0 & 4,0 & 0,0 & 24,0 & 22,0 & 11,0 & 57,0 & 1,1 & 154,0 & 111,0 \\
\hline P2.2 & 119,0 & 26,0 & 69,0 & 65,0 & $<20$ & 4,0 & 0,0 & 21,0 & 22,0 & 10,0 & 54,0 & 1,0 & 144,0 & 116,0 \\
\hline $\mathrm{P} 2.3$ & 120,0 & 27,0 & 74,0 & 73,0 & 24,0 & 5,0 & 0,0 & 25,0 & 21,0 & 11,0 & 52,0 & 1,1 & 153,0 & 113,0 \\
\hline Média & 121,7 & 26,3 & 70,7 & 70,0 & 24,0 & 4,3 & 0,0 & 23,3 & 21,7 & 10,7 & 54,3 & 1,1 & 150,3 & 113,3 \\
\hline DP & 3,8 & 0,6 & 2,9 & 4,4 & 0,0 & 0,6 & 0,0 & 2,1 & 0,6 & 0,6 & 2,5 & 0,1 & 5,5 & 2,5 \\
\hline P3.1 & 63,0 & 44,0 & 111,0 & 66,0 & 21,0 & $<3$ & 0,1 & 25,0 & $<8$ & 12,0 & 15,0 & 2,1 & 272,0 & 122,0 \\
\hline P3.2 & 56,0 & 43,0 & 105,0 & 58,0 & 21,0 & $<3$ & 0,1 & 20,0 & $<8$ & 12,0 & 15,0 & 2,1 & 268,0 & 122,0 \\
\hline P3.3 & 62,0 & 40,0 & 103,0 & 61,0 & 22,0 & $<3$ & 0,1 & 22,0 & $<8$ & 12,0 & 16,0 & 1,9 & 229,0 & 113,0 \\
\hline Média & 60,3 & 42,3 & 106,3 & 61,7 & 21,3 & - & 0,1 & 22,3 & - & 12,0 & 15,3 & 2,0 & 256,3 & 119,0 \\
\hline DP & 3,8 & 2,1 & 4,2 & 4,0 & 0,6 & - & 0,0 & 2,5 & - & 0,0 & 0,6 & 0,1 & 23,8 & 5,2 \\
\hline P4.1 & 53,0 & 48,0 & 135,0 & 54,0 & 21,0 & $<3$ & 0,1 & 20,0 & $<8$ & 11,0 & 13,0 & 2,3 & 285,0 & 137,0 \\
\hline $\mathrm{P} 4.2$ & 51,0 & 51,0 & 139,0 & 54,0 & 21,0 & $<3$ & 0,1 & 18,0 & $<8$ & 11,0 & 14,0 & 2,5 & 289,0 & 151,0 \\
\hline P4.3 & 49,0 & 46,0 & 137,0 & 51,0 & $<20$ & $<3$ & 0,1 & 18,0 & $<8$ & 10,0 & 12,0 & 2,2 & 273,0 & 138,0 \\
\hline Média & 51,0 & 48,3 & 137,0 & 53,0 & 21,0 & - & 0,1 & 18,7 & - & 10,7 & 13,0 & 2,3 & 282,3 & 142,0 \\
\hline DP & 2,0 & 2,5 & 2,0 & 1,7 & 0,0 & - & 0,0 & 1,2 & - & 0,6 & 1,0 & 0,2 & 8,3 & 7,8 \\
\hline P5.1 & 70,0 & 49,0 & 73,0 & 85,0 & 28,0 & 5,0 & 0,1 & 26,0 & $<8$ & 17,0 & 17,0 & 2,3 & 310,0 & 152,0 \\
\hline P5.2 & 68,0 & 50,0 & 69,0 & 82,0 & 28,0 & 5,0 & 0,1 & 22,0 & $<8$ & 16,0 & 17,0 & 2,4 & 313,0 & 156,0 \\
\hline P5.3 & 70,0 & 52,0 & 71,0 & 144,0 & 29,0 & 5,0 & 0,1 & 24,0 & $<8$ & 17,0 & 17,0 & 2,5 & 321,0 & 153,0 \\
\hline Média & 69,3 & 50,3 & 71,0 & 103,7 & 28,3 & 5,0 & 0,1 & 24,0 & - & 16,7 & 17,0 & 2,4 & 314,7 & 153,7 \\
\hline DP & 1,2 & 1,5 & 2,0 & 35,0 & 0,6 & 0,0 & 0,0 & 2,0 & - & 0,6 & 0,0 & 0,1 & 5,7 & 2,1 \\
\hline
\end{tabular}

DP: Desvio padrão calculado para 3 medidas. Os elementos Cd (<0,3mg/kg), Mo (<3mg/kg), Sb (<10mg/kg), Sn $(<20 \mathrm{mg} / \mathrm{kg})$ e W $(<20 \mathrm{mg} / \mathrm{kg})$ estão fora do limite de detecção instrumental.

alteração das rochas da Bacia do Paraná (análises detalhadas podem ser obtidas em Piccirillo \& Melfi, 1998) reduz a contribuição geogênica (valores de background regional são baixos).

Os tributários exercem influência na concentração total de contaminantes no rio principal (Förstner et al., 2004) mas isto não ocorre no córrego Liso e rio Uberabinha. No Córrego Liso (Pontos 3 e 4) apesar de drenar o distrito industrial, não apresentou nos sedimentos coletados, as maiores concentrações médias totais de elementos tóxicos. A erosão dos solos é um problema ambiental que ocorre de forma intensiva na bacia e particularmente nesta área. Seu resultado na qualidade dos sedimentos do rio Uberabinha parece ser divergente. Por um lado, no caso dos solos agrícolas, as partículas transportadas e depositadas nas margens podem conter resíduos de agroquímicos adsorvidos nos colóides. Este mecanismo de contaminação de corpos hídricos é bastante conhecido. Por outro lado, a deposição recente de partículas de solo limpo pode minimizar ou mesmo ocultar concentrações químicas em sedimentos mais antigos e, conseqüentemente, mais profundos. Sedimentos não contaminados podem se misturar com sedimentos contaminados e resultar em decréscimo na- 
tural do nível de contaminação (SedNet 2007). A retirada da vegetação de cerrado e, principalmente, da mata ciliar neste segmento do córrego contribuíram para a intensa erosão lateral das margens, causando assoreamento e formação de bancos em diversos segmentos dos rios. A deposição de sedimentos relativamente "limpos" recobriu áreas de inundação e influenciou nos resultados de concentração dos elementos tóxicos. Desta forma, evidencia-se a necessidade do controle da erosão na bacia para também controlar a qualidade dos sedimentos. No Ponto 4 destaca-se o elevado teor de $\mathrm{Cr}$ que pode estar relacionado à décadas de lançamento de efluente de curtume do distrito industrial. Embora essa atividade tenha cessado atualmente, os efeitos no ambiente persistem.

Finalmente no Ponto 5 a jusante, embora tenham sido determinadas as maiores concentrações médias de $\mathrm{Co}, \mathrm{Cu}, \mathrm{Sc}$, Ti e V, não foi observado efeito acumulativo, sugerindo uma mistura de poluentes como reflexo de fontes difusas. Poluição difusa de caracteriza pela presença crônica de uma mistura de elementos químicos tóxicos sobre uma ampla superfície de área cujos valores excedem os limites regulatórios de qualidade ambiental (Posthuma et al., 2008).

Implicações ambientais Os resultados obtidos apresentaram grandes variações na distribuição, na concen- tração e no tipo de elementos químicos tóxicos fixados nos sedimentos recentemente depositados em diferentes segmentos do rio Uberabinha.

As evidências de ocorrência de impactos relacionados à concentração de elementos tóxicos ao meio ambiente e aos organismos no rio Uberabinha se pautaram nos critérios para avaliação da qualidade dos sedimentos estabelecidos pela Resolução CONAMA 344/04 (Tab. 4). O limite mínimo - TEL (Threshold Effect Level) ou Nível 1 - representa a concentração abaixo da qual raramente são esperados efeitos adversos aos organismos. O limite máximo - PEL (Probable Effect Level) ou Nível 2 - representa a concentração acima da qual é freqüentemente esperado o citado efeito adverso para os organismos. Na faixa entre os Níveis 1 e 2 situam-se os valores onde ocasionalmente esperam-se tais efeitos. São considerados os elementos $\mathrm{As}, \mathrm{Cd}, \mathrm{Pb}, \mathrm{Cu}, \mathrm{Cr}$, Ni e Zn. O cenário recente revelou concentração em níveis acima do aceitável para $\mathrm{Pb}, \mathrm{Cu}$, $\mathrm{Cr}$, Ni e Zn. Os elementos As e Cd não estão presentes ou estão fora do limite de detecção do método. Todos os elementos detectados estão acima do nível mínimo aceitável (Nível 1) em algum ponto de coleta enquanto as concentrações de $\mathrm{Cr}$, Ni e Zn ultrapassam o máximo aceitável (Nível 2). Estes resultados, ainda que iniciais, evidenciam a possibilidade de ocorrências de toxidez e efeitos adversos ao ambiente e saúde humana.

Tabela 4 - Tabela com os valores estabelecidos pela Resolução CONAMA 344/04 e valores obtidos nos 5 pontos de análise do Rio Uberabinha (Uberlândia, MG).

\begin{tabular}{llllllll}
\hline Elementos & $\begin{array}{l}\text { Valores orientadores } \\
(\mathrm{mg} / \mathrm{kg})\end{array}$ & \multicolumn{5}{c}{$\begin{array}{c}\text { Valores } \\
\text { médios determinados } \\
(\mathrm{mg} / \mathrm{kg})\end{array}$} \\
\hline & $\begin{array}{l}\text { TEL } \\
(\text { Nível 1) }\end{array}$ & $\begin{array}{l}\text { PEL } \\
(\text { Nível 2) }\end{array}$ & Ponto 1 & Ponto 2 & Ponto 3 & Ponto 4 & Ponto 5 \\
\hline $\mathrm{As}$ & 5,9 & 17 & n.d. & n.d. & n.d. & n.d. & n.d. \\
\hline $\mathrm{Cd}$ & 0,6 & 3,5 & n.d. & n.d. & n.d. & n.d. & n.d. \\
\hline $\mathrm{Pb}$ & 35 & 91,3 & $\mathbf{5 6 , 7}$ & 21,7 & n.d. & n.d. & n.d. \\
\hline $\mathrm{Cu}$ & 35,7 & 197 & $\mathbf{7 5}$ & $\mathbf{7 0}$ & $\mathbf{6 1 , 7}$ & $\mathbf{5 3}$ & $\mathbf{1 0 3 , 7}$ \\
\hline $\mathrm{Cr}$ & 37,3 & 90 & $\mathbf{1 0 5 , 7}$ & $\mathbf{7 0 , 7}$ & $\mathbf{1 0 6 , 3}$ & $\mathbf{1 3 7}$ & $\mathbf{7 1}$ \\
\hline $\mathrm{Ni}$ & 18 & 35,9 & $\mathbf{4 0 , 3}$ & $\mathbf{2 3 , 3}$ & $\mathbf{2 2 , 3}$ & $\mathbf{1 8 , 7}$ & $\mathbf{2 4}$ \\
\hline $\mathrm{Zn}$ & 123 & 315 & 41,3 & $\mathbf{1 3 3 , 3}$ & 74 & 72,7 & 81 \\
\hline
\end{tabular}

${ }^{1}$ CONAMA (2004); ${ }^{2}$ Rio Uberabinha; n.d.= não determinado

CONCLUSÕES O aumento da concentração de elementos tóxicos no ambiente é uma conseqüência da urbanização e da industrialização (Adriano et al., 2004). Os contaminantes fixados nos sedimentos, a partir de certo nível, implicam em riscos para o ambiente e qualidade das águas (Salomons \& Brils 2004).

Apesar de esta pesquisa ser considerada piloto e contar, ainda, com baixa densidade de pontos de amostragem na bacia, os primeiros resultados obtidos em pontos representativos do rio evidenciaram condições críticas na qualidade dos sedimentos recentemente depositados no rio Uberabinha, excedendo os valores orientadores mínimos de qualidade estabelecidos pelo CONAMA (2004). Nos cinco pontos de amostragem, determinaram-se valores elevados de $\mathrm{Pb}, \mathrm{Cu}, \mathrm{Cr}, \mathrm{Ni}$ e Zn. Estes elementos aparecem como prioridade nos estudos ambientais pois são dominantes em resíduos das atividades econômicas, desde a mineração até indústrias. Outros elementos também considerados tóxicos como, por exemplo, As, Cd, Sb e Sn, não aparecem na lista de elementos com valores orientadores, porém podem ser considerados como tóxicos para plantas e 
animais, mesmo em baixas concentrações, quando formam compostos organo-metálicos (Lerche et al., 2004; Fernandez et al., 2005). Amparados no conceito de que todos os elementos têm importância no funcionamento dos ciclos biogeoquímicos ambientais, o uso de técnicas multi-elementares nas pesquisas ambientais deve ser privilegiada por permitir a quantificação de um grande número de elementos químicos (Markert \& Friese, 2000). É importante salientar que as informações sobre as concentrações de massa ou total não são suficientes para determinação da biodisponibilidade dos elementos tóxicos (Adriano et al., 2004) mas elas se configuram como um excelente critério para definição da extensão da concentração, tendo como referência os valores orientadores estabelecidos para manutenção da qualidade e multifuncionalidade do sedimento e ambiente aquático (Sterckeman et al., 1996; Gleyses et al., 2002). Este procedimento permite que se estabeleça uma rede de coleta e monitoramento da qualidade dos sedimentos. Alguns elementos-traço são essenciais para a nutrição das plantas $(\mathrm{B}, \mathrm{Cu}, \mathrm{Fe}, \mathrm{Mn}, \mathrm{Mo}, \mathrm{Zn})$, animais ( $\mathrm{As}, \mathrm{Cu}, \mathrm{Co}, \mathrm{Fe}, \mathrm{Mn}, \mathrm{Mo}, \mathrm{Zn}, \mathrm{Cr}, \mathrm{F}, \mathrm{Ni}, \mathrm{Se}$, $\mathrm{Sn}, \mathrm{V}$ ) e humana (neste caso omitem-se As e V). Outros
$(\mathrm{Cd}, \mathrm{Hg}, \mathrm{Pb})$ não têm valor nutricional mas importância ambiental. Quando estes elementos estão presentes nos organismos em valores acima de background, podem ocasionar sérias doenças especialmente pela não biodegradação e por se acumularem nos ciclos biogeoquímicos globais (Adriano et al., 2004).

Pelos resultados obtidos neste trabalho, é evidente que a urbanização e a agricultura têm grande influência no transporte e acúmulo de substâncias potencialmente tóxicas nos sedimentos do rio Uberabinha. Por se caracterizar como fontes difusas, há uma maior dificuldade em identificar as áreas fontes, em controlar e mitigar os efeitos da poluição (Posthuma et al., 2008). $\mathrm{O}$ controle da qualidade ambiental, a identificação e monitoramento das fontes de poluição podem ser feitos por meio dos estudos de sedimentos, sendo desejável incorporá-los nos programas de planejamento e gestão dos recursos hídricos.

Agradecimentos Nossos sinceros agradecimentos à Fundação de Amparo à Pesquisa do Estado de Minas Gerais (FAPEMIG, Projeto CRA1768/06) pelo financiamento desta pesquisa.

\section{Referências}

Adriano D.C., Wenzel W.W., Vangronsveld J., Bolan N.S. 2004. Role of assisted natural remediation in environmental cleanup. Geoderma, 122:121-142.

Ahlf W. \& Förstner U. 2001. Managing contaminated sediments. Part I: Improving chemical and biological criteria. J. Soils \& Sediments, 2(1):30-36.

Alloway B.J. 1990. The origins of heavy metals in soils. In: Alloway B.J. (ed.) Heavy metls in soils. New York, John Wiley, p. 29-39.

Angelotti-Neto A., Crestana S., Oliveira S.C., Barbosa R.V.R. 2004. Metais pesados provenientes da atividade agrícola: formas, prevenção e controle. In: Espindola E. \& Wendland E. (orgs.) Bacia hidrográfica: diversas abordagens de pesquisa. Série Ciências da Engenharia Ambiental. São Carlos, Editora RiMa, v.3, p.1-16.

Brismar A. 2002. River systems as providers of good and services: a basis for comparing desired and undesired effects of large dam projects. Env. Man., 29:598-609.

Campos M.L., Silva F.N., Furtini Neto A.E., Guilherme L.R.G., Marques J.J., Antunes A.S. 2005. Determinação de cádmio, cobre, cromo, níquel, chumbro e zinco em fosfatos de rocha. Pesquisa Agropecuária Brasileira, 40(4):361-367.

Carneiro, R.M.A. 2004. Bioindicadores vegetais de poluição atmosférica: uma contribuição para a saúde da comunidade. Dissertação de Mestrado, Universidade de São Paulo, Ribeirão Preto, 146p.

CONAMA. Conselho Nacional do Meio Ambiente. Resolução 344/04. Disponível em http//www.mma.gov.br. Acessado em 01 de nov de 2007.

Dai J.J., Lorenzato S., Rocke D.M. 2004. A knowledge-based model of watershed assessment for sediment. Environmental Modelling \& Software, 19:423-473.
Duarte R.P.S. \& Pasqual A. 2000. Avaliação da quantidade de cádmio $(\mathrm{Cd})$, chumbo $(\mathrm{Pb})$, Níquel $(\mathrm{Ni})$ e zinco $(\mathrm{Zn})$ em solos, plantas e cabelos humanos. Energia na Agricultura, 15(1):46-58.

Fernandez M.A., Wagener A.L.R., Limaverde A.M., Scofield A.L., Pinheiro F.M., Rodrigues E. 2005. Imposex and surface sediment speciation: a combined approach to evaluate organotion contamination in Guanabara Bay, Rio de Janeiro, Brazil. Marine Environmental Research, 59:435-452.

Förstner U. 2003. Geochemical techniques on contaminated sediments - river basin view. Part I: Integrated water quality management: river basin approach. Environ. Sci. \& Pollut. Res., 10(1):58-62.

Förstner U. 2004. Traceability of sediment analysis. Trends in Analytical Chemistry, 23(3):217-236.

Förstner U, Heise S., Schwartz R., Westrich B., Ahlf W. 2004. Historical contaminated sediments and soils at the river basin scale. Examples from the Elbe River catchment area. J. Soils \& Sediments, 4(4):247-260.

Gleyzes C., Tellier S., Astruc M. 2002. Fractionation studies of trace elements in contamineted soils and sediments: a review of sequential extraction procedures. Trends in Analytical Chemistry, 21(6-7): 451-467.

Hissler C., Bortoletto Jr. M.J., Mortatti J., de Oliveira H., Probst J.L. 2004. Heavy metals (Ni, Zn, Cu, Pb, Cd) bioavailability potential and speciation in the Tietê river bottom sediments, Brazil. In: International Symposium Environmental Geochemistry in Tropical Countries, $4^{\text {th }}$, Buzios, R.J., Brazil, Book of Abstracts, p. 555-556.

INSTITUTO MINEIRO DE GESTÃO DAS ÁGUAS (IGAM). 2005. Qualidade das águas Superficiais no Estado de Minas Gerais, Projeto Águas de Minas. Relató- 
rio de Monitoramento das Águas Superficiais na Bacia do Rio Paranaíba em 2004. Disponível em http//www. igam.mg.gov.br/index.php. Acessado em 11 jan 2006.

Kasper D., Nogueira S., Palermo E.F.A., Branco C.W.C., Malm O. 2004. Mercury in bottom sediment from Vigário Reservoir, Brazil. In: International Symposium Environmental Geochemistry in Tropical Countries, $4^{\text {th }}$, Buzios, R.J., Brazil, Book of Abstracts, p. 558-560.

Lerche D., Matsuzaki S.Y., Sorensen P.B., Carlsen L., Nielsen O.J. 2004. Ranking of chemical substances based on Japanese pollutant release and transfer register using partial order theory and randon linear extensions. Chemosphere, 55(7):1005-1025.

Markert B., Friese K. 2000. Trace elements. Their distribution and effects in the environment. Amsterdan, Elsevier Science, 324p.

Miguel A.H. 1992. Poluição atmosférica urbana no Brasil: uma visão geral. Química Nova, 15(2):116-125.

Minella J.P.G., Merten G.H., Reichert J.M., Santos D.R.dos. 2007. Identificação e implicações para a conservação do solo das fonts de sedimentos em bacias hidrográficas. $R$. Bras. Ci. Solo, 31:1637-1646.

Mozeto A.A., Silvério P.F., DePaula F.C.F., Bevilacqua J.E., Patella E., Jardim W.F. 2003. Weakly-bound metals and total nutrient concentration of bulk sediments from some water reservoir in São Paulo State, SE Brazil. In: Manavar M. (ed.) Sediment Quality Assessment and Management Insight and Progress. Ecovision World Monograph Series.

Mudrock A. \& MacKnight S.D. 1994. Techniques for aquatic sediments sampling. $2^{\text {nd }}$, FL, USA., Ed. Publishers, Boca Raton.

Nriagu, J.O. \& Pacyna, J.M. 1988. Quantitative assessment of worldwide contamination of air, water and soils with trace elements. Nature, 333:134-139.

Owens P.N. 2005. Conceptual models and budgets for sediment management at the River Basin Scale. J. Soils \& Sediments, 5(4):201-212.

Piccirillo E.M. \& Melfi A.J. 1988. The Mesozoic flood volcanism of the Parana Basin: petrogenetic and geophysical aspects. São Paulo, Instituto Astronômico e Geofísico Publisher, University of São Paulo, 600p.

Posthuma L., Eijsachers H.J.P., Koelmans A.A., Vijver M.G. 2008. Ecological effects of diffusion pollution are sitespecific and require high-tier risk assessment to improve site management decisions: a discussion paper. Science of the Total Environment, 406(3):503-517.
Rosolen V.; Herpin U., Fränzle S., Breulmann G., Camargo P.B., Paganini W.S., Cerri C.C., Melfi A.J., Markert B.A. 2005. Land application of wastewater in Brazil - a scientific challenge: chemical characterization of soil at Populina, São Paulo State. Journal of Soils and Sediments, 5(2):112-120.

Salomons W. 2005. Sediments in the catchment-coast continuum. J. Soils \& Sediments, 5(1):2-8.

Salomons W. \& Förstner U. 1984. Metals in the hidrocycle. Berlin, Springer-Verlag, 349p.

Salomons W. \& Brils J. 2004. Contaminated sediments in European River Basins. In:___ (eds). SedNet booklet as final report for the ECFP5 Thematic Network Project SedNet. Disponível em: http://www.sednet.org/download/Sednet booklet final.pdf. Acesso em: 10/04/2008.

EUROPEAN SEDIMENTT NETWORK (SedNet). 2007. Sediment management - an essential element of river basin management plans. In: Report on the SedNet round table discussion. Venice, 22-23 november 2007, 28p. Disponível em: http://www.sednet.org/ download/061122_Report_SedNet_Round_Table_Discussion.pdf. Acesso em: 07/03/2008.

Singh M., Ansari A.A., Müller G., Singh I.B. 1997. Heavy metals in freshly deposited sediments of the Gomati River (a tributary of the Ganga River): effects of human activities. Environ. Geol., 29(3-4): 246-252.

Sterckeman T., Gomez A., Ciesielski H. 1996. Soil and waste analysis for environmental risk assessment in France. The Science of the Total Environment, 178:63-69.

Stigliani W.M. 1988. Changes in valued "capacities" of soil and sediments as indicators of non-linear and time-delayed environmental effects. Environ. Monit. Assess., 10:245-307.

Wilcke W., Lifienfein J., Lima S.C., Zech W. 1999. Contamination of highly weathered urban soils in Uberlândia, Brazil. J. Plant Nutr. Soil Sci., 162:539-548.

Robaina L.E., Formoso M.L., Pires C.A.da F. 2002. Metais pesados nos sedimentos de corrente como indicadores de risco ambiental - Vale do Rio dos Sinos, RS. Revista do Instituto Geológico, 23(2):35-47.

Vörösmarty C.J., Meybeck M., Fekete B., Sharma K., Green P., Syvitski J.P.M. 2003. Anthropogenic sediment retention: major global impact from registered river impoundments. Glob. Planet Change., 39:169-190.

Manuscrito ID 11484

Submetido em 12 de junho de 2008 Aceito em 11 de março de 2009 